will contribute to existing literature and theory on community based The Flint Community's Action Plan to Rebuild Trust and Encourage Resiliency During the Post Water Crisis Phase Karen D. Calhoun ${ }^{1}$, Kent Key, PhD ${ }^{3}$, E. Yvonne Lewis ${ }^{2}$, Susan J Woolford ${ }^{1}$, E. Hill DeLoney ${ }^{4}$, Jennifer Carerra, $\mathrm{PhD}^{3}$, Joe Hamm, PhD ${ }^{3}$, Ella Greene-Moton ${ }^{4}$, Patricia Piechowski ${ }^{1}$, Kaneesha Wallace ${ }^{5}$ and Elder Sarah Bailey, $\mathrm{PhD}^{4}$ ${ }^{1}$ University of Michigan School of Medicine; ${ }^{2}$ National Center for African American Health Consciousness; ${ }^{3}$ Michigan State University; ${ }^{4}$ Community Based Organization Partners and ${ }^{5}$ Healthy Flint Research Coordinating Center

OBJECTIVES/SPECIFIC AIMS: o To review the community's recommendations on how to rebuild trust in the Flint community. o To review effective community engagement strategies utilized with the Flint Special Projects for project conceptualization, participant recruitment, data analysis, project oversight, and dissemination. METHODS/STUDY POPULATION: The study population includes nearly two hundred residents representing seniors, youth and diverse ethnicities recruited to participate in eleven focus group meetings. The population also represents the general public who attended informational meetings in Flint, Michigan to learn about the crisis and allow residents to voice their opinions and concerns during the onset of the crisis. The project is a mixed methods community based participatory research effort that utilized community decision making in all phases of the effort such as pre-conception, implementation, dissemination and advocacy to encourage the community's recommendations are adopted at policy and institutional responsiveness levels. It includes three community engaged research efforts: (project 1) A qualitative analysis of community sentiment provided during 17 recorded legislative, media and community events, and (projects 2-3) two mixed methods efforts utilizing purposive sampling of stakeholders whose voice may not have been heard. RESULTS/ ANTICIPATED RESULTS: The project presents a qualitative analysis of the community's voice during the onset of the man-made disaster when the community first became aware of the emergency manager's plans to switch the water source. It also reflects current perspectives of community voice since the projects are scheduled to end late February 2019. Findings from a trust measure administered to nearly two hundred residents will be presented, along with a qualitative analysis of focus group findings among segments of the population (seniors, youth, and diverse ethnicities) who may have been left out of narratives on the water crisis. Finally, the project will compare empowerment and resiliency approaches being utilized in Flint, Michigan to recover from the disaster with other approaches grounded in literature and theory. DISCUSSION/SIGNIFICANCE OF IMPACT: Communities of color often experience social determinants of health which negatively impact their health, well-being and human rights. Some Flint citizens are experiencing negative health consequences (i.e., rashes, brain and behavioral sequelle, fertility, etc.) as a result of the disaster, and are uncertain of health outcomes in the future. This is the first project to rigorously document and analyze levels of trust and mistrust in the city of Flint since the water disaster occurred. The qualitative research will guide future clinical research that will benefit this traumatized community experiencing high levels of mistrust (i.e., government, elected officials, etc.). The community engaged methodology involved residents and study participants in all phases of the project including project oversight, validating and analyzing data, and dissemination. This methodology and citizen science. The approaches empowered a call to action among residents, for example, seniors who attended two senior focus group sessions shared "they are hopeful and have a purpose," resulting in the creation of a council (with officers) at their housing complex to advocate for the well-being of seniors during the recovery process. Recruitment methodologies were extremely successful due to resident level trust in community leaders and community partner organizations. Finally, the project's examination of approaches encouraging empowerment and resiliency will provide lessons learned for other communities challenged with crisis.

\title{
The Spectrum of Homelessness and Its Association with Maternal Morbidity
}

Kelley N. Robinson ${ }^{1}$, Kelly Bower, PhD, MSN/MPH, RN ${ }^{1}$, Jennifer Stewart, PhD, $\mathrm{RN}^{1}$, Nancy Perrin, $\mathrm{PhD}^{1}$,

Nancy Glass, PhD, MPH, RN, FAAN ${ }^{1}$, Keshia Pollack-Porter, PhD, $\mathrm{MPH}^{1}$ and Phyllis Sharps, PhD, RN, FAAN ${ }^{1}$

${ }^{1}$ Johns Hopkins University

OBJECTIVES/SPECIFIC AIMS: To examine maternal morbidity and its related social determinants among women experiencing homelessness during pregnancy. METHODS/STUDY POPULATION: This study will use an exploratory sequential mixed method design to explore and examine the structural, interpersonal and individual factors contributing to maternal morbidity among a convenience sample of 150 English speaking women experiencing homelessness during a pregnancy within the last 3 years in Baltimore. In the qualitative phase of the study, we will conduct semi-structured interviews with 15 women purposively sampled to refine the relationships between resilience, social determinants of health and multilevel factors that impact maternal morbidities. Factors of interest include prenatal care received, barriers and facilitators to receiving prenatal care, maternal morbidities, social support, and strategies used to manage their condition during this time. Using the findings from the qualitative phase, a quantitative survey will be developed to gather data on topics that emerged in the interviews. In addition, the Housing Instability Index will be used to measure the degree of homelessness as defined by the degree of housing instability in a 6-month period. Using the 25-item Connor-Davidson Resilience Scale, resilience levels among women in the sample will be assessed as a moderating factor in the examination of the relationship between a pregnant woman's homeless status and maternal morbidity. Descriptive statistics and logistical regression tests will be used to analyze these relationships while controlling for other structural, interpersonal, and individual factors that may be associated with maternal morbidity. RESULTS/ANTICIPATED RESULTS: Qualitatively we expect to gain insight into the relationship between the extrinsic and intrinsic factors impacting maternal morbidities and the health behaviors and practices used by women to manage their pregnancy while homeless. These findings will inform the quantitative survey development and help generalize the quantitative findings. We expect to identify the common morbidities in this population we anticipate that there will be differences in maternal morbidity among the different types of homelessness. Maternal morbidity will be higher among women with a greater degree of homelessness. Resilience will have a moderating effect on the relationship between homelessness and maternal 